\title{
Diabetes Mellitus Causes Early Ultrastructural Changes to the Nuclei and Mitochondria of Neurons and Astrocytes in Rats Subjected to a Brief Period of Cerebral Ischemia
}

\author{
C. Ding*, Q.P. He**, and P.A. Li* \\ *Department of Cell Molecular Biology and Medicine, **Biological Electron \\ Microscopy Facility, University of Hawaii, Honolulu, HI 96822
}

It has been previously demonstrated that hyperglycemia caused by glucose infusion or diabetes enhances neuronal damage induced by transient global or focal cerebral ischemia [1]. In addition to neurons, astrocytes may also be the target and damage to the astrocytes may in turn influence the neuronal survival [2]. The objective of the present study was to define the ultrastructural alterations of neurons and astrocytes in streptozotocin-induced diabetic rats subjected to a brief period of global ischemia.

Five minutes of global ischemia was induced in non-diabetic and diabetic rats. Brain samples were collected after $30 \mathrm{~min}, 6 \mathrm{~h}, 1,3$, and 7 days of recirculation as well as from sham-operated controls. Electron microscopy demonstrated homogenous neuronal nuclear chromatin, visible nuclei and intact nuclear membranes up to $1 \mathrm{~d}$ of recovery in both non-diabetic and diabetic rats. Mitochondrial morphology was normal up to $6 \mathrm{~h}$ of recovery, but disarray of mitochondrial cristae, mild lucency and swelling were observed in a few mitochondria after $1 \mathrm{~d}$ of recovery in non-diabetics. In contrast, diabetic rats showed disarray of mitochondrial cristae after $30 \mathrm{~min}$ and developed more severe swelling after $6 \mathrm{~h}$ and $1 \mathrm{~d}$ of recovery. These findings are consistent with our previous published ultrastructural studies in rats subjected to an intermediate period of global ischemia [3]. No apoptotic bodies were observed in any of the sections examined. In astrocytes, the nuclear chromatin was homogenous, nuclei were visible, and nuclear membrane was intact up to $1 \mathrm{~d}$ of recovery in cortex. Mitochondrial morphology was normal up to $1 \mathrm{~d}$ of recovery in nondiabetic rats. Compare to nondiabetic rats, nuclear and mitochondrial morphological alterations were prominent in diabetic rats after 1 day of recovery. Nuclear shrinkage, chromatin condensation and void space were evident. Disarray of mitochondrial cristae, lucency, and swelling were observed in astrocytes after $1 \mathrm{~d}$ of recovery.

The results suggest that diabetic hyperglycemia causes damage to both neurons and astrocytes in early reperfusion phase. The damage to mitochondria in neurons and astrocytes may activate mitochondria-initiated cell death pathways results in DNA fragmentation and ischemic cell death.

\section{References}

[1] P.A. Li and B.K. Siesjö, Acta Physiol Scand, 161 (1997) 567.

[2] Y. Chen and R.A. Swanson, J Cereb Blood Flow Metab. 23 (2003), 137.

[3] P.A. Li et al., J Cereb Blood Flow Metab, 21 (2001) 568.

[4] This study was supported by NIH/NCRR (RR16453, RR03061) and Ingeborg v. F. Mckee Fund of Hawaii Community Foundation to PAL. 
A

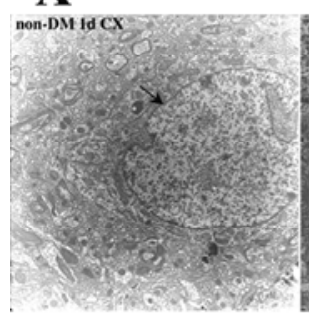

B

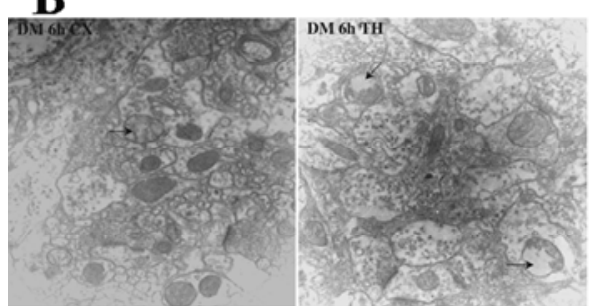

C
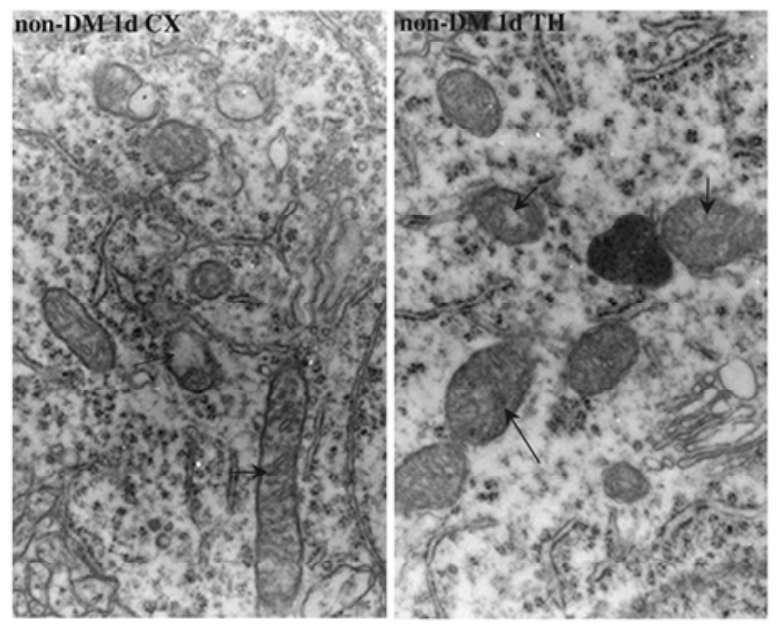

Figure 1. Electron micrographs of nuclei and mitochondria of neurons in the neocortex (CX) and thalamus (TH) in non-diabetic (non-DM) and diabetic (DM) rats subject to five minutes global ischemia. $\mathbf{A}$, nuclear morphology in $\mathrm{CX}$ in both nonDM and DM rats after $1 \mathrm{~d}$ recovery, magnification was $8,000 \mathrm{X}$. Arrows denote nuclei. $\mathbf{B}$, mitochondrial morphology in $\mathrm{CX}$ and $\mathrm{TH}$ in DM rats after $6 \mathrm{~h}$ recovery, magnification was 16,000X. C, mitochondrial morphology in CX and TH in non-DM rats after $1 \mathrm{~d}$ recovery, magnification was $10,000 \mathrm{X}$. Arrows denote mitochondria with disarrayed cristae, mild lucency and swelling.
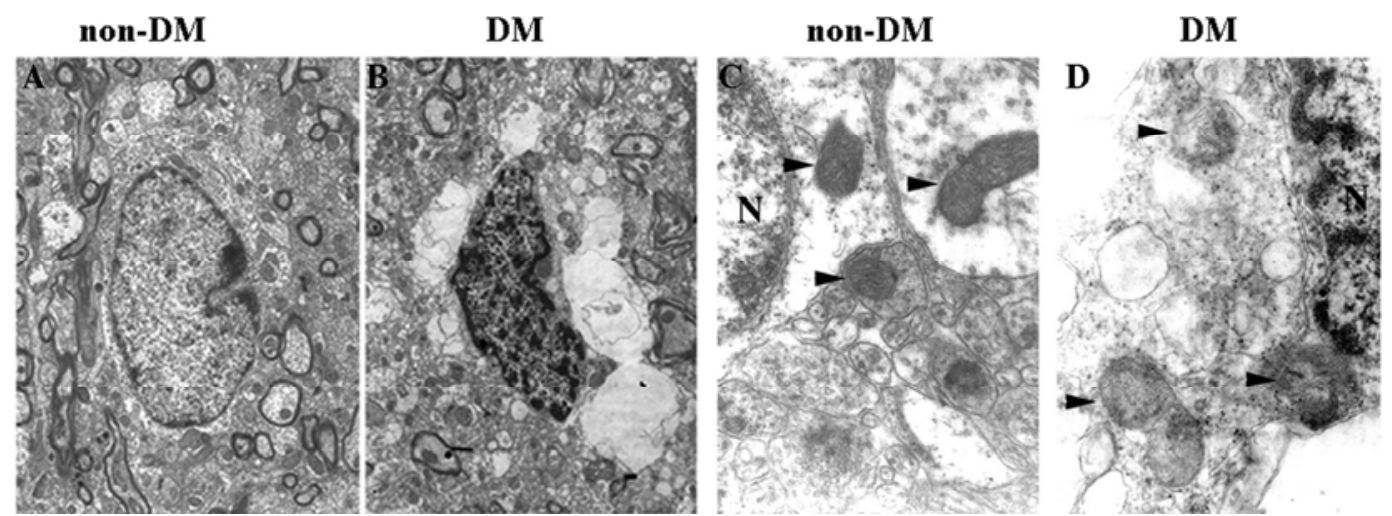

Figure 2. Electron micrographs of nuclei and mitochondria of astrocytes in nondiabetic (non-DM) and diabetic (DM) rats subject to five minutes global ischemia, followed $1 \mathrm{~d}$ of recirculation. Nuclear shrinkage, chromatin condensation and void space around the astrocytes are evident in diabetics (A) but not in non-diabetics (B). Magnification=2,000x. Mitochondrial lucency and swelling are observed in diabetic (C) but not in non-diabetic animals (D). Magnification $=40,000 \mathrm{X}$. Arrows denote mitochondria with disarrayed cristae, mild lucency and swelling. 